\title{
Current and future role of echocardiography in arrhythmogenic right ventricular dysplasia/cardiomyopathy
}

Thomas P. Mast, Arco J. Teske, Pieter A. Doevendans, Maarten J. Cramer

Department of Cardiology, Division Heart, University Medical Center, Utrecht, The Netherlands

\begin{abstract}
Arrhythmogenic right ventricular dysplasia/cardiomyopathy $(A R V D / C)$ is an inherited progressive cardiomyopathy, clinically characterized by ventricular arrhythmias and increased risk of sudden cardiac death. Echocardiography has a role in the diagnosis and prognosis of $A R V D / C$. However, in the current era of magnetic resonance imaging (MRI), the role of echocardiography in ARVD/C patients and family member screening is subject to debate. Relatively novel echocardiographic techniques, such as three-dimensional right ventricular $(3 D-R V)$ imaging and tissue deformation imaging, may improve the diagnostic and prognostic performance of echocardiography in these patients.

$3 D-R V$ imaging provides more insights on $R V$ anatomy and global function compared to conventional echocardiography. Subtle $R V$ regional wall motion abnormalities, and mechanical dyssynchrony, are accurately measured by tissue deformation imaging.

Several studies suggest an incremental value of novel echocardiographic parameters in addition to conventional measurements. Moreover, new parameters indicating subtle RV dysfunction, and mechanical dyssynchrony, are of predictive value and could help in risk stratification of $A R V D / C$ patients.

New robust parameters, derived from $3 D-R V$ echocardiography and $R V$ tissue deformation imaging, in combination with established conventional parameters, suggest that there is a current and future role for echocardiography in ARVD/C supplementing MRI. (Cardiol J 2015; 22, 4: 362-374)
\end{abstract}

Key words: ARVD/C, echocardiography, tissue deformation imaging, three-dimensional echocardiography, prognosis, diagnosis

Editorial p. 355

\section{Introduction}

Arrhythmogenic right ventricular dysplasia/ /cardiomyopathy (ARVD/C) is an inherited progressive cardiomyopathy, clinically characterized by life-threatening ventricular arrhythmias and, in advanced stages, heart failure [1]. In up to $60 \%$ of ARVD/C patients, genetic mutations are found in genes encoding for desmosomal proteins $[2,3]$. Desmosomes are located in the intercalated disc and provide structural and functional integrity between cardiac myocytes [4]. Desmosomal dysfunction leads to both mechanical and electrical uncoupling $[4,5]$. This mechanical uncoupling is

Address for correspondence: Thomas P. Mast, MD, University Medical Center, Heidelberglaan 100, 3584CX, Utrecht, The Netherlands, e-mail: T.P.Mast@umcutrecht.nl 


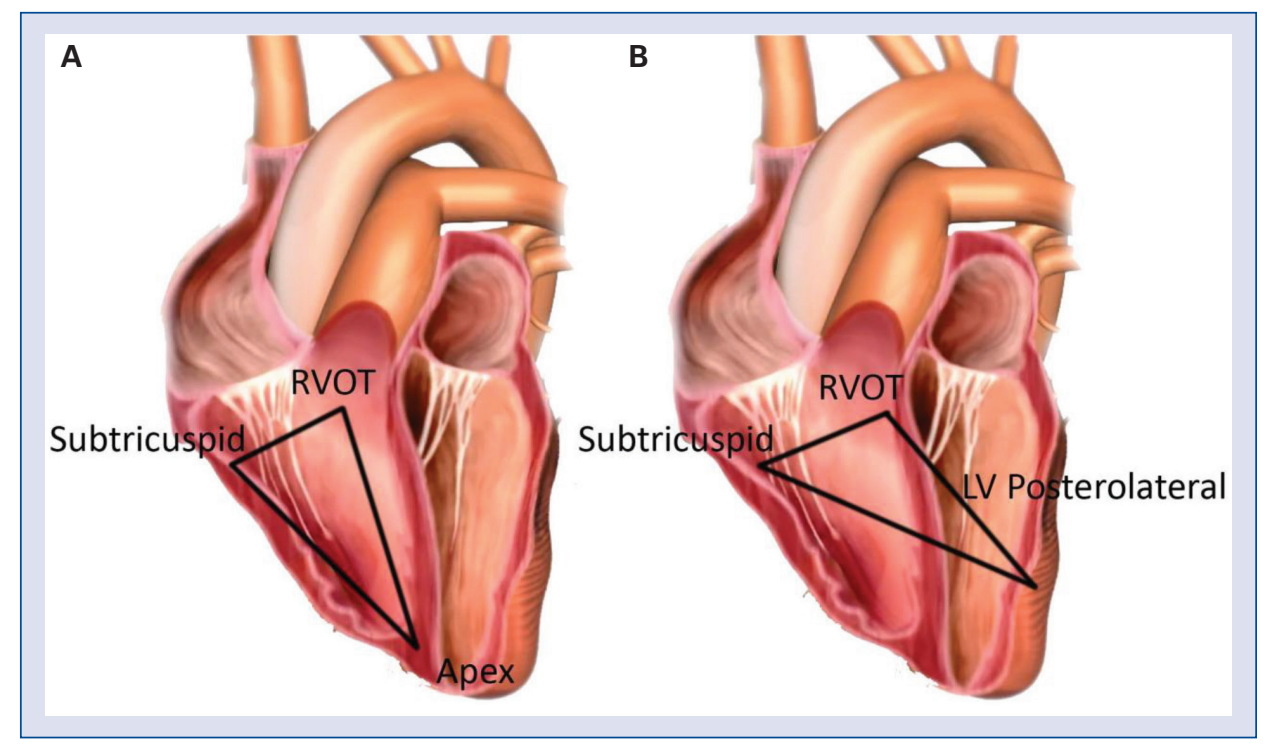

Figure 1. Triangle of dysplasia. A. Original triangle of dysplasia consisting of the following regions: 1) subtricuspid region, 2) right ventricular outflow tract (RVOT), and 3) apical region; B. Revised triangle of dysplasia. The left ventricular (LV) posterolateral wall seems to be a clear predilection site of arrhythmogenic right ventricular dysplasia/cardiomyopathy and is more commonly involved compared to the apical region.

associated with fibro-fatty replacement of the myocardium and right ventricular (RV) dysfunction [6]. Electrical uncoupling of cardiac myocytes and the presence of surviving myocardial bundles within the fibrofatty tissue promote activation delay, therefore providing a substrate (macro re-entry) for ventricular arrhythmias [5, 7].

In Europe and North America, desmosomal mutations are predominantly found in the plakophilin-2 (PKP2) gene $[3,8,9]$. However, pathogenic ARVD/C mutations show incomplete penetrance and wide variable phenotypic expression [3]. Therefore, risk stratification remains challenging. Although desmosomes are expressed in all cardiac myocytes, a typical distribution of disease expression is observed. Typical involved areas are the subtricuspid region, the right ventricular outflow tract (RVOT), and the left ventricular (LV) posterolateral wall [10]. Traditionally, the RV apex was seen as an important region which was involved in this disease and was included in the so called "triangle of dysplasia" [1]. However, recent studies have shown that the RV apex is only involved in advanced stages of the disease [10]. Secondly, $\mathrm{LV}$ involvement is more common than previously thought, with a clear predilection site in the LV posterolateral region (Fig. 1) [10, 11].

$\mathrm{ARVD} / \mathrm{C}$ is diagnosed according to major and minor criteria stated in the 2010 revised Task Force criteria (TFC) [12]. The $2010 \mathrm{TFC}$ include different categories: (1) structural RV alterations, (2) tissue characterization, (3) depolarization and repolarization abnormalities, (4) ventricular arrhythmias, and (5) family history and genetic data [12]. The diagnostic criteria are fulfilled by the presence of 2 major, or 1 major plus 2 minor criteria or 4 minor criteria from different groups. RV functional and structural alterations are visualized by using different imaging modalities such as echocardiography, magnetic resonance imaging (MRI), and angiography. Diagnostic imaging criteria are scored if a regional $\mathrm{RV}$ wall motion abnormality is present in combination with RV dilatation or global RV systolic dysfunction [12]. Modality-specific cut-off values concerning global RV dysfunction and RV dilatation are provided in the 2010 TFC (Table 1) [12].

Echocardiography is a noninvasive, and widely available imaging tool to evaluate patients with known or suspected ARVD/C. However, complete and accurate RV echocardiography is difficult due to the retrosternal position and complex geometry of the RV. MRI is not hampered by these limitations and has therefore taken a prominent role in imaging for ARVD/C [13]. Recently, MRI was found to be of superior value for the diagnosis of ARVC compared to conventional echocardiography [14]. Nevertheless, MRI is also prone to false-positive findings if there is an overreliance on detection of intramyocardial fat and wall thinning [15]. In addition, determination of normal RV wall motion 
Table 1. 2010 Task Force criteria. Global or regional dysfunction and structural alterations.

\begin{tabular}{l} 
Echocardiography \\
Regional RV akinesia, dyskinesia, or aneurysm and \\
one of the following parameters: \\
Major \\
PLAX RVOT $\geq 32 \mathrm{~mm}$ or corrected for BSA $\geq 19 \mathrm{~mm} / \mathrm{m}^{2}$ \\
PSAX RVOT $\geq 36 \mathrm{~mm}$ or corrected for BSA $\geq 21 \mathrm{~mm} / \mathrm{m}^{2}$ \\
RV-FAC $\leq 33 \%$ \\
Minor \\
PLAX RVOT $\geq 29$ to $<32 \mathrm{~mm}$ or corrected \\
for BSA $\geq 16$ to $<19 \mathrm{~mm} / \mathrm{m}^{2}$ \\
PSAX RVOT $\geq 32$ to $<36 \mathrm{~mm}$ or corrected \\
for BSA $\geq 18$ to $<21 \mathrm{~mm} / \mathrm{m}^{2}$ \\
RV-FAC $>33 \%$ to $\leq 40 \%$ \\
Magnetic resonance imaging \\
Regional RV akinesia, dyskinesia, or dyssynchronous \\
RV contraction \\
Major \\
Ratio of RVEDV to BSA $\geq 110 \mathrm{~mL} / \mathrm{m}^{2}$ (male) \\
or $\geq 100 \mathrm{~mL} / \mathrm{m}^{2}$ (female) \\
RVEF $\leq 40 \%$ \\
Minor \\
Ratio of RVEDV to BSA $\geq 100$ to $<110 \mathrm{~mL} / \mathrm{m}^{2}$ (male) \\
or $\geq 90$ to $<100 \mathrm{~mL} / \mathrm{m}^{2}$ (female) \\
RVEF $>40 \%$ to $\leq 45 \%$ \\
RV angiography \\
Major \\
Regional RV akinesia, dyskinesia, or aneurysm \\
Minor \\
No minor criterion is specified for this modality \\
\hline
\end{tabular}

Derived from the 2010 Task Force criteria; RVOT — right ventricular outflow tract; FAC — fractional area change; RV — right ventricle: RVEF - right ventricular ejection fraction; RVEDV — right ventricular end-diastolic volume; PLAX/PSAX - parasternal long/shorts axis; BSA - body surface area

around the moderator band insertions remains difficult by MRI $[15,16]$.

Emerging new echocardiographic techniques, specifically three dimensional (3D) RV echocardiography and tissue deformation imaging, may increase the performance of echocardiography, overcoming the previously mentioned shortcoming of conventional RV echocardiography $[17,18]$. $3 \mathrm{D}$ imaging enables more accurate RV volumetric measurements compared to conventional echocardiography $[19,20]$. Regional deformation imaging, another relatively new technique, quantifies regional $\mathrm{RV}$ wall motion and provides timing of regional motion $[18,21]$.

Implantable cardioverter-defibrillators (ICD) are frequently implanted in $\mathrm{ARVD} / \mathrm{C}$ patients to prevent sudden arrhythmic death. These metallic devices make MRI unsuitable for evaluation of ARVD/C patients. For the purpose of monitoring $\mathrm{ARVD} / \mathrm{C}$ patients, echocardiography is the current preferred imaging technology [22]. Novel echocardiographic techniques could conceivably provide new predictors for the occurrence of cardiac events during follow-up in addition to conventional measures of RV and LV function.

In this review, we aim to evaluate the current and future role of both conventional and new echocardiographic parameters in $\mathrm{ARVD} / \mathrm{C}$ with respect to the diagnosis of the disease as well as follow-up. We provide illustrative examples of this relatively rare disease entity and we have added our echocardiographic protocol as a guide for the clinician.

\section{Conventional echocardiography}

Extensive fibrofatty replacement of the myocardium affects regional ventricular wall motion and global systolic function [1]. Impaired systolic function leads to structural RV remodeling and is detected by conventional echocardiography as both global RV dilatation and RV systolic dysfunction [23, 24].

Regional and global RV dilatation are well described phenotypic features in ARVD/C patients [24]. RV inflow tract, RVOT, and longitudinal dimensions have been found to be increased compared to healthy controls (Fig. 2) [17, 23-26]. Several robust parameters representing RV systolic function, such as the tricuspid annular plane systolic excursion (TAPSE), peak systolic RV annular velocity, and RV-fractional area change (RV-FAC), provide insight into global RV function and are generally decreased in ARVD/C (Fig. 3) [17, 23-25, 27]. Altered morphologic appearances, e.g. a hyper-reflective moderator band and excessive trabecular derangement, are also reported in ARVD/C (Table 2) [24]. However, these findings lack specificity and were not included in the 2010 TFC. A study performed by Yoerger et al. [24] in 2005 found that impaired RV-FAC and RVOT dilatation were the most common abnormal echocardiographic findings in ARVD/C patients. RVOT dilatation in the parasternal long-axis view was present in all ARVD/C patients and decreased RV-FAC was present in $62 \%$ of patients. Eventually, these parameters were chosen for the echocardiographic 2010 TFC [12]. TAPSE and peak systolic RV annular velocity were not evaluated in this study and could be the reason why these parameters are not part of the 2010 TFC. 


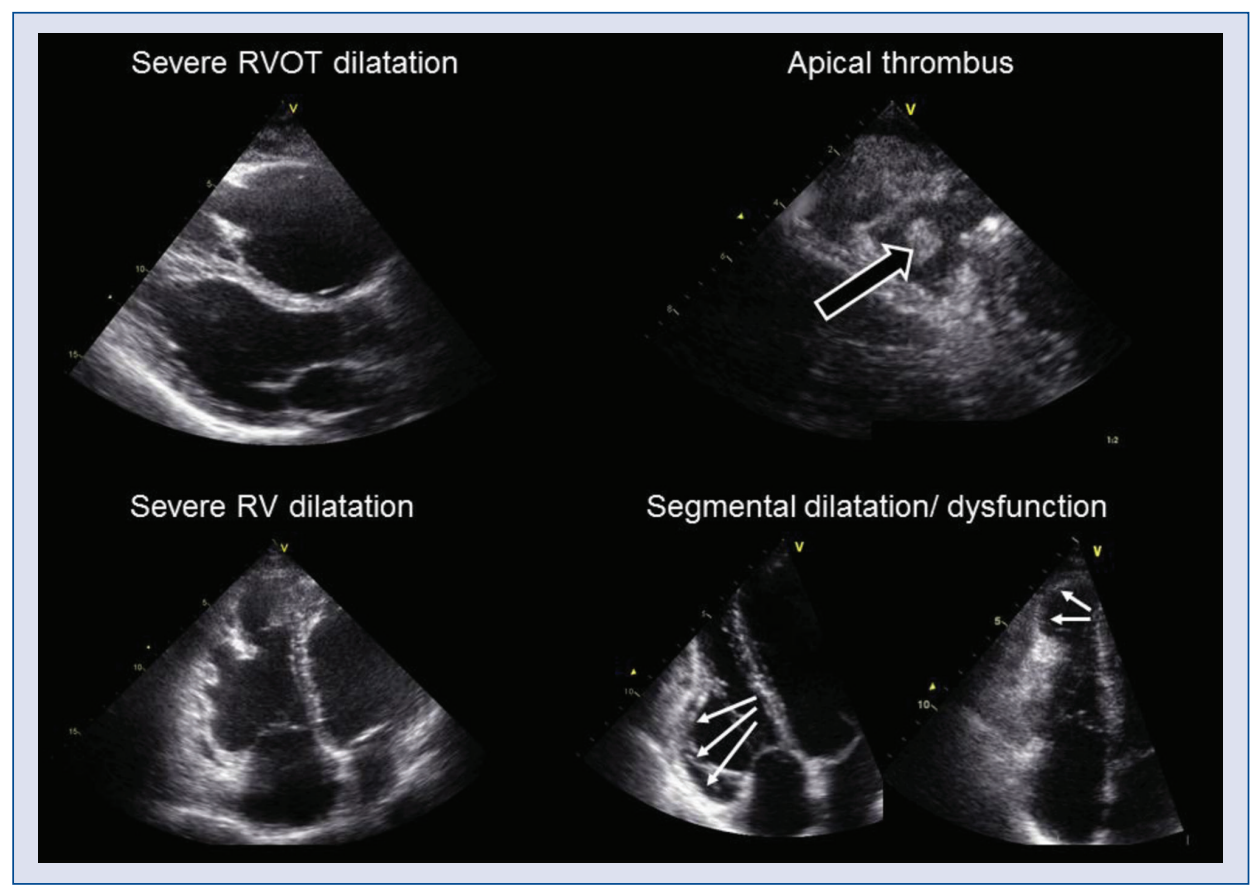

Figure 2. Conventional echocardiography in an arrhythmogenic right ventricular dysplasia/cardiomyopathy (ARVD/C) patient; Left upper: PLAX, severe RVOT dilatation $\left(57 \mathrm{~mm}, 27.4 \mathrm{~mm} / \mathrm{m}^{2}\right)$ in 57 -year-old ARVD/C patient; Right upper: RV SAX, apical thrombus in 16-year-old ARVD/C patient, RV-FAC was 29\%; Left lower: AP4CH, severe dilatation of RV, RVIT = $49 \mathrm{~mm}$, and hyperreflective moderator band; Right lower: AP4CH, apical aneurysm and segmental subtricuspid dilatation; PLAX — parasternal long axis view; RVOT — right ventricular outflow tract; RV — right ventricle; SAX — short axis view; RV-FAC — right ventricular-fractional area change; AP4CH — apical 4 chamber view; RVIT — right ventricular inflow tract.

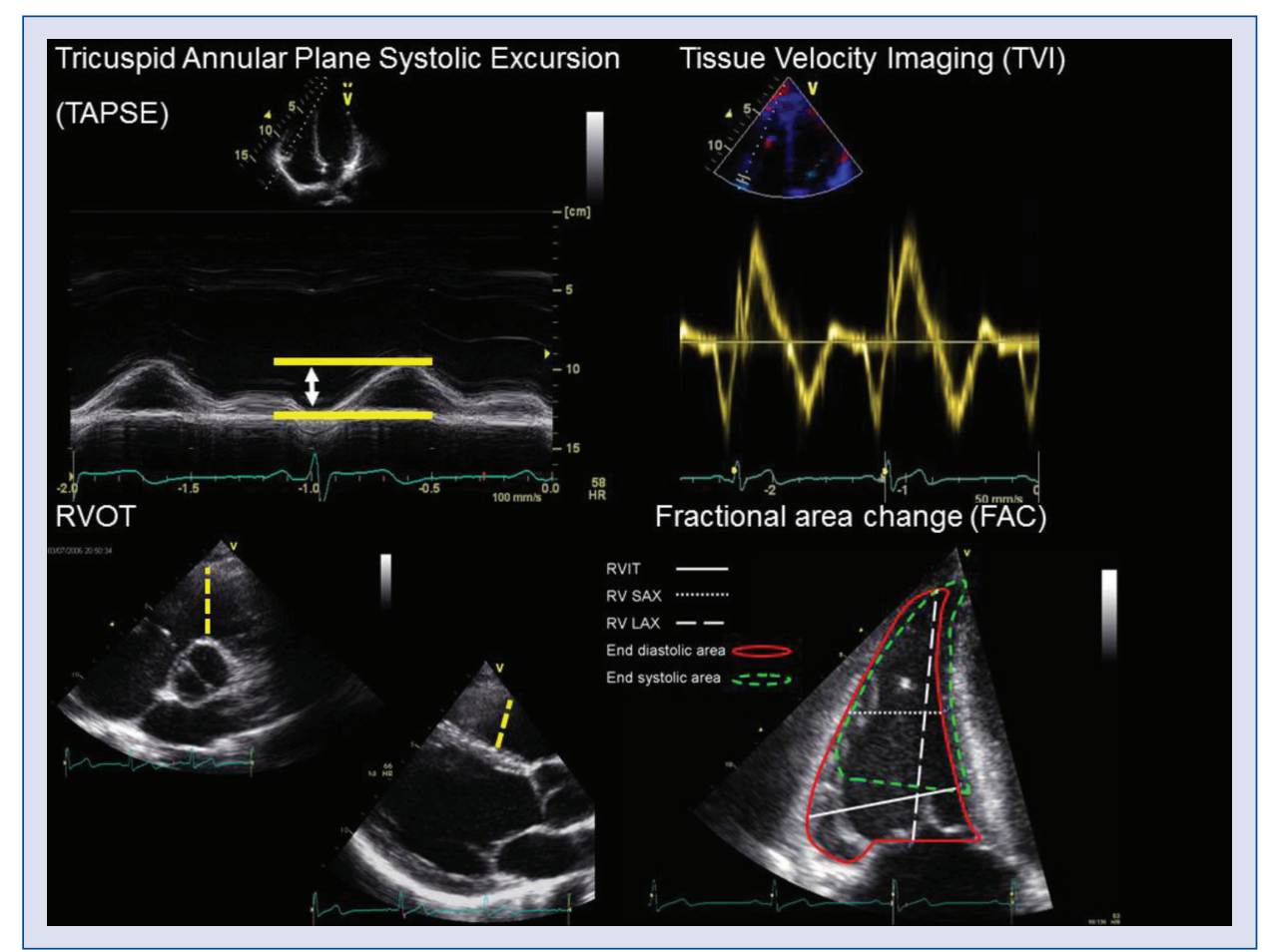

Figure 3. Conventional right ventricular measurements. Example of measurements of tricuspid annular plane systolic excursion (TAPSE) (upper left), peak annular systolic velocity (upper right), right ventricular outflow tract (RVOT) (lower left) and right ventricular-fractional area change (RV-FAC) (lower right). 
Table 2. Wall motion abnormalities and morphological appearances in arrhythmogenic right ventricular dysplasia/cardiomyopathy patients.

\begin{tabular}{lc}
\hline Parameters & N $=29$ [24] \\
\cline { 2 - 2 } & Percent of probands \\
\hline RV global systolic function & 38 \\
RV-FAC $\geq 32 \%$ & 28 \\
RV-FAC $\geq 26 \%$ to $<32 \%$ & 34 \\
RV-FAC $<26 \%$ & 79 \\
RV regional WMA & 45 \\
RVOT & 55 \\
Anteroseptal & 70 \\
Anterior & 72 \\
Apex & 55 \\
Septal & 59 \\
Inferior basal & 52 \\
Inferior apical & 31 \\
Hyperreflective & \\
moderator band & 54 \\
Excessive/abnormal & 17 \\
trabeculations & \\
Sacculations & \\
\hline RV & \\
\hline
\end{tabular}

RV — right ventricle; RV-FAC — right ventricular-fractional area change; RVOT — right ventricular outflow tract; WMA — wall motion abnormalities

Conventional echocardiography is part of the current diagnostic $2010 \mathrm{TFC}$ and consists of visual regional wall motion analyses, outflow tract dimensions and RV-FAC [12]. An echocardiographic TFC is assigned if RVOT dilatation or decreased RV-FAC is observed in combination with abnormal regional wall motion [12]. Akinetic, dyskinetic, or aneurysmatic RV wall segments are considered abnormal. Importantly, hypokinesis is not a part of the 2010 TFC [12]. In a study performed in our institution, we found that regional hypokinesia is a common finding in healthy individuals [28]. Depending on the degree of RVOT dilatation and reduction of RV-FAC, a major or minor criterion is assessed (Table 1) [12]. The presence of abnormal regional wall motion is mandatory for a major or minor criterion regardless of RVOT dilatation and/or systolic dysfunction. Therefore, optimal visual RV wall motion analysis is crucial and we suggest that all RV regions should be visualized during the examination (Fig. 4) [26, 29]. Contrast enhanced echocardiography could be used to improve delineation of the RV free wall and enables more accurate RV wall motion analyses where there is suboptimal imaging quality [30]. Values for echocardiographic TFC were chosen for high specificity $(76-95 \%)$ resulting in reduced sensitivity $(55-87 \%)[12,14]$. Abnormal findings not fulfilling the current TFC should either be further assessed by MRI or these patients should undergo a follow-up echocardiogram after 1 year. This recommendation is based on our current practice and experience since there is currently no literature to support it. In addition to the diagnostic role of conventional echocardiography in ARVD/C, the prognostic value of echocardiography in ARVD/C has been extensively explored [22, 31, 32]. Both decreased TAPSE and RV-FAC are associated with the occurrence of major adverse cardiac events in ARVD/C [22, 32]. Both parameters are easily obtained and aid risk stratification of ARVD/C patients (Fig. 3). Several studies have shown the clinical importance of LV involvement in ARVD/C [11, 31, 32]. Although, originally considered an end-stage phenomenon, LV involvement in a broad spectrum of ARVD/C patients has been shown $[10,33,34]$. Echocardiographically measured reduced $L V$ ejection fraction has been found to be the strongest independent predictor of adverse outcome [31, 32].

All aforementioned parameters are easily obtained by conventional echocardiography and provide useful information during diagnostic evaluation and follow-up in ARVD/C. The precise interval of repeated echocardiographic evaluation is unknown in patients diagnosed with $\mathrm{ARVD} / \mathrm{C}$. We advise echocardiographic interrogation in patients when clinical changes occur (e.g. heart failure, arrhythmias, and syncope). In stable patients with no change in any clinical parameter, an echocardiographic follow up every $4-5$ years seems reasonable (unpublished data).

We have provided the ARVD/C focused echocardiographic protocol which is currently in use in our institution (Fig. 4).

\section{Three-dimensional echocardiography}

There is a marked difference between MRI and echocardiography in the 2010 TFC with respect to measuring RV dilatation and RV systolic dysfunction. Global RV dilatation by MRI is directly measured by RV end-diastolic volume (RVEDV), whereas conventional echocardiography only uses segmental outflow tract dilatation [12]. RVEDV and RV end-systolic volume (RVESV) leads to a 3D measurement of RV systolic function 


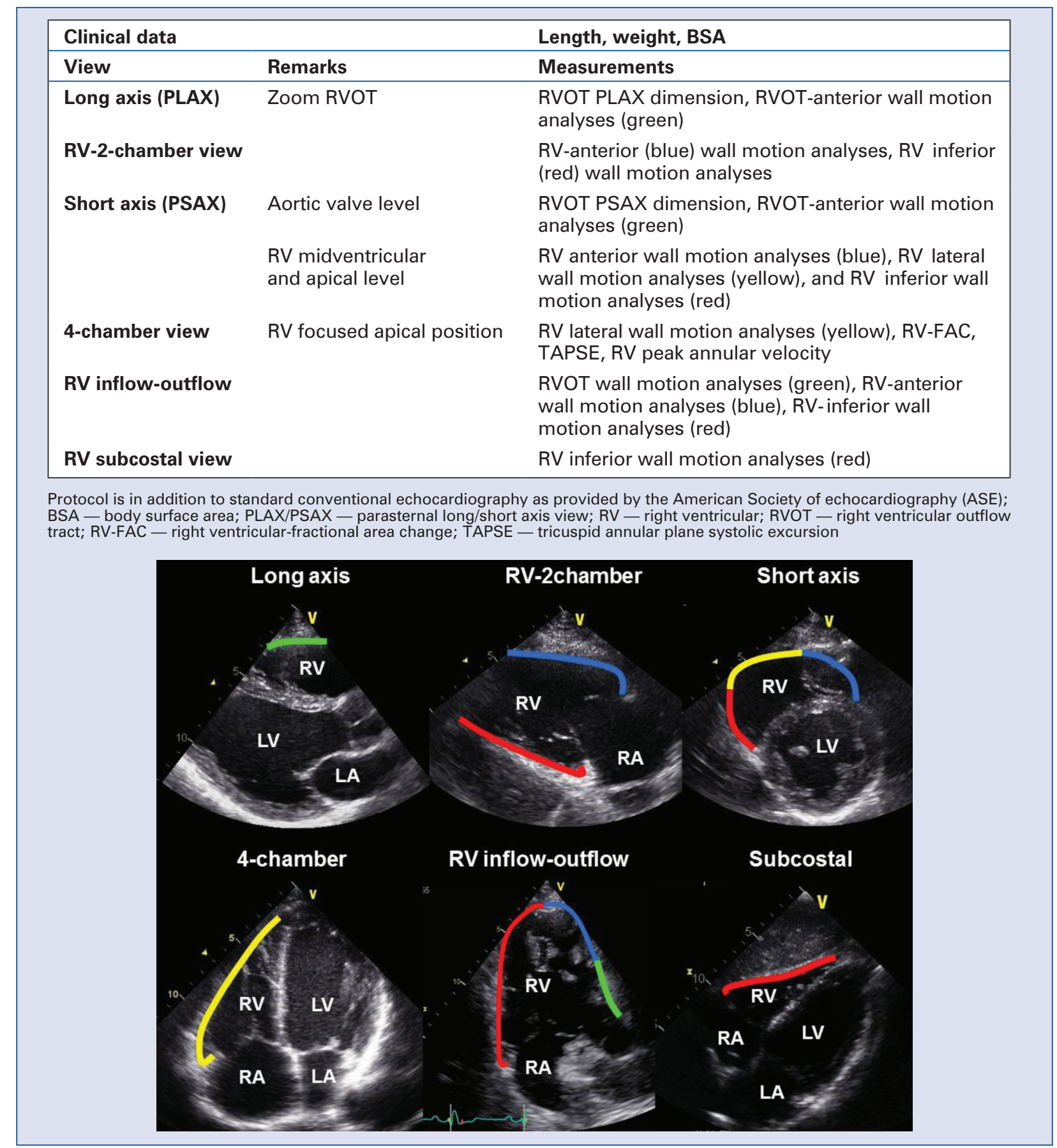

Figure 4. Echocardiographic arrhythmogenic right ventricular dysplasia/cardiomyopathy (ARVD/C) protocol and right ventricular (RV) view; RA — right atrium; LV — left ventricle; LA — left atrium.

(ejection fraction) by MRI. Echocardiographic RV systolic function only contains a 2D measurement, by measuring the amount of RV area decrease during systole. This limited approach is due to the retrosternal location and complex crescent geometry of the RV. Therefore, the simple geometrical assumptions based on 2D acquisition that is frequently used in assessing LV dimen- sions, are invalid for the RV and prevents optimal volumetric calculation. These measurements by echocardiography are likely inferior to the 3D volumetric analysis performed by MRI. However, $3 \mathrm{D}$-echocardiography is both feasible and comparable to MRI with respect to RV volumes and RV ejection fraction (RVEF) (Fig. 5) [19, 20,35]. With the implementation of 3D-echocardiography, subtle 


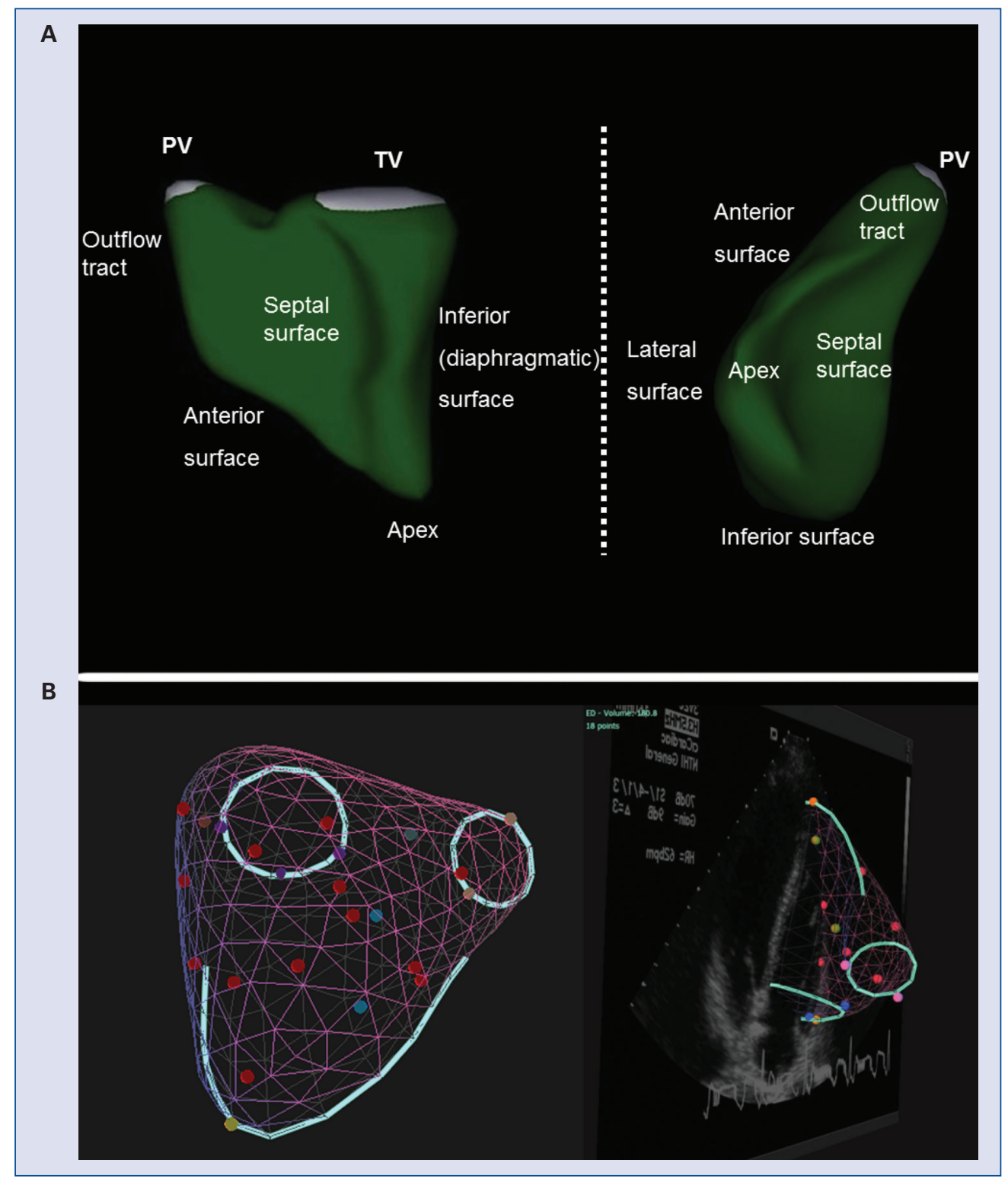

Figure 5. Perimeters of 3-dimensional-right ventricular (3D-RV) of imaging; A. 3D-RV echocardiographic imaging providing excellent insight in RV anatomy; B. 3D acquisition with knowledge based reconstruction. Multiple landmarks, chosen in 2D images, are used to reconstruct the RV anatomy; PV — pulmonary valve; TV — tricuspid valve.

global RV systolic dysfunction can be seen in the early stages of ARVD/C, and can contribute to early diagnosis [36]. In ARVD/C patients, Prakasa et al. [19] found varying results by $2 \mathrm{D}$-echocardiography compared to MRI for RVESV, RVEDV and RVEF with Pearson's correlations of $0.72,0.50$ and 0.88 , respectively. 3D-echocardiography is hampered by acoustic dropout of the RV free wall and the anterior RV wall in patients with a dilated RV [19]. The current software algorithms may account for the frequently observed abnormal RV shape in ARVD/C patients with large saccular aneurysms. These two mechanisms likely explain the low yield of $3 \mathrm{D}$-echocardiography in this subset of patients.
A possible approach to overcome the limitation of acoustic dropout is to use 3D-based RV reconstruction to obtain RV volumes and function (Fig. 5) $[37,38]$. The reconstruction technique uses a magnetic field generator and a magnetic field sensor attached to the transducer to accurately localize $2 \mathrm{D}$ obtained RV anatomic landmarks within the magnetic field [37]. The reconstruction algorithm uses the distances between RV landmarks to match the landmarks to a catalog of subjects with similar $\mathrm{RV}$ anatomy. This 3D-based technique shows high correlation with MRI derived volumetric measurements in patients with pulmonary hypertension and congenital heart diseases. Inter-method correlation 


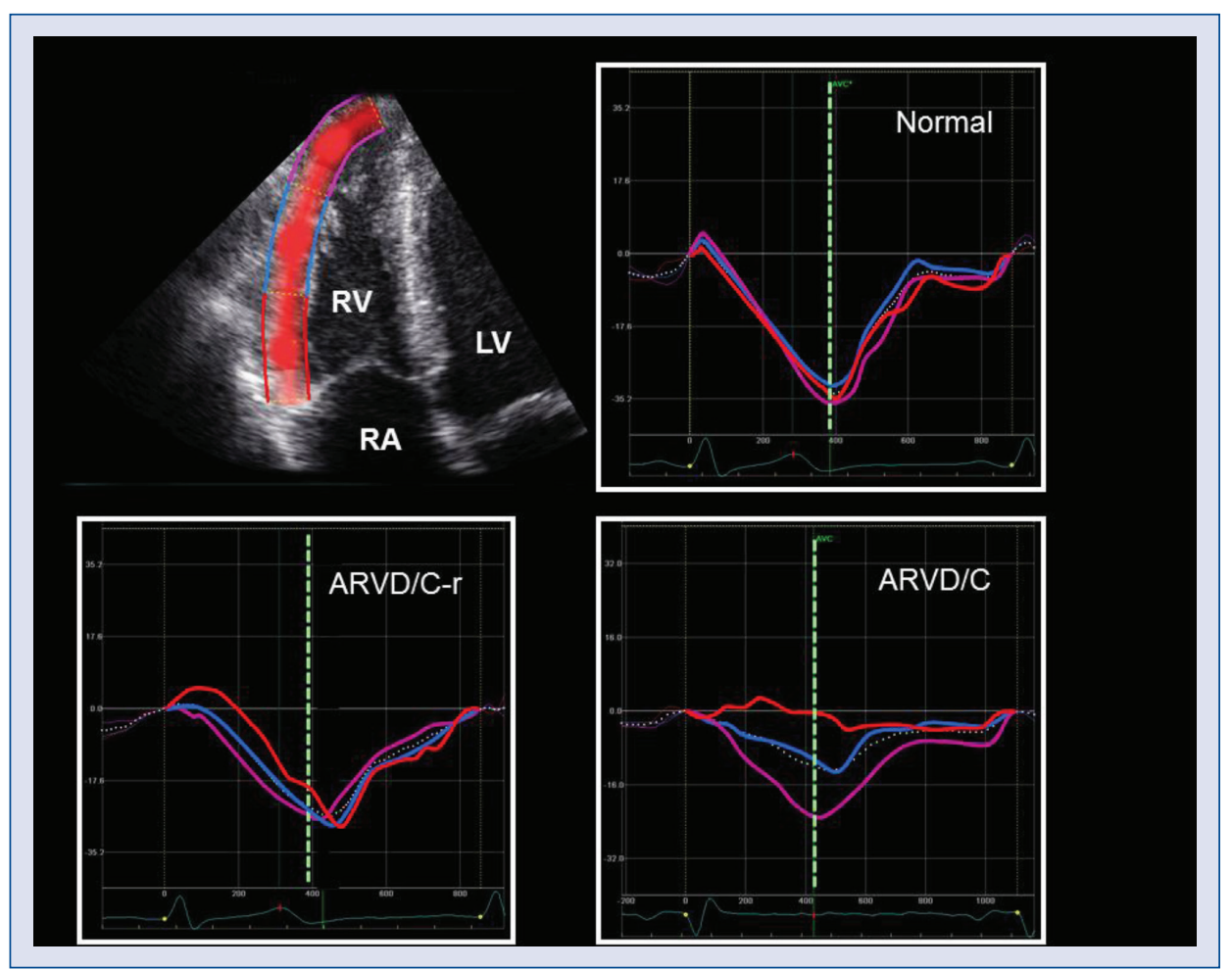

Figure 6. Tissue deformation imaging in arrhythmogenic right ventricular dysplasia/cardiomyopathy (ARVD/C); Left upper: Tissue deformation imaging by speckle tracking. In the apical 4 chamber view the right ventricular (RV) lateral wall is traced and divided into the basal (red), mid (blue), and apical view (pink); Right upper: Normal RV deformation patterns with shortening during systole and stretching in diastole. All systolic strain values are approximately $-30 \%$. Cut off value is $-18 \%$ to differentiate between normal and abnormal peak systolic strain; Left lower: RV deformation pattern in an ARVD/C relative. The apical (pink) and mid segment (blue) shows normal deformation. However, the basal (red) curve showed marked prestretch, delayed onset of shortening, and post-systolic shortening. In this patient, abnormal subtricuspid deformation was seen in absence of structural abnormalities according to the 2010 revised Task Force criteria; Right lower: RV deformation pattern in an ARVD/C patient. The apical segment (pink) shows normal deformation. Mid segment shows lower values of systolic peak strain $(<-18 \%)$ and post-systolic shortening. The basal segment shows a dyskinetic pattern with stretching during systole; LV — left ventricular; RA — right atrium.

was 0.87-1.00 (RVEDV), 0.88-1.00 (RVESV), and 0.75-0.88 (RVEF) [37, 38]. We currently lack studies with 3D-based $\mathrm{RV}$ reconstruction in $\mathrm{ARVD} / \mathrm{C}$ patients.

\section{Tissue deformation imaging}

Echocardiographic tissue deformation imaging is a relatively new technique that provides quantitative regional wall motion analysis. Currently, two different techniques are commercially available: tissue Doppler imaging and speckle-tracking. Both techniques rely on different principles to calculate both regional and global deformation and deformation rate. We have previously shown that both techniques are feasible in the RV and that the calculated values are comparable to one another in $\mathrm{ARVD} / \mathrm{C}$ patients [18].
Tissue Doppler imaging uses the differences between two velocity-time curves to measure the regional deformation of the myocardium. Velocitytime curves are derived from two points of known distance and encompass a region of interest. Deformation of the region of interest is absent if the difference between two velocity-time curves is zero, indicating this net resultant force on the myocardium is zero. However, differences in velocitytime curves indicate deformation, i.e. shortening or lengthening, of the myocardium. Deformation is plotted against time in a strain-time curve over the cardiac cycle (Fig. 6).

Speckle tracking (or 2D-strain) uses natural acoustic echocardiographic reflection (speckles) in the B-mode image made within the myocardium to measure deformation. This speckle pattern is unique for each myocardial region and it is 
relatively stable throughout the cardiac cycle. The displacement of this speckled pattern is considered to follow myocardial movement and the change between speckles represents myocardial deformation. With dedicated software, it is possible to accurately track these speckle patterns throughout the cardiac cycle. The resulting data provide both regional and global deformation (rate) in the longitudinal direction. Radial strain in the thin walled RV is unreliable and we do not recommend its use in the RV $[18,39]$. $\mathrm{An}$ in depth review on image acquisition, post processing, interpretation and implementation of both techniques has been published [18].

Due to the longitudinal fiber orientation of the $\mathrm{RV}$ cardiomyocytes, the resulting RV systolic wall motion is predominantly longitudinal [40]. Thus, the base of the ventricle (annular plane) moves apically, while the active inward motion is minimal and predominantly a result of tethering/traction at the LV insertion sites [41]. Consequently, RV myocardial tissue deformation results in longitudinal systolic shortening (Fig. 5). The RV lateral free wall recordings, acquired in the apical 4 chamber view, are feasible for deformation imaging $(>90 \%)$ in $\mathrm{ARVD} / \mathrm{C}$ patients $[17,42,43]$. Limitations in temporal resolution and angle dependency exclude the anterior, inferior, and RVOT for optimal deformation imaging analysis. However, Greiner et al. [43] explored multi-plane strain imaging to assess the antero- and infero-lateral side of the RV free wall and reported a high feasibility $(84 \%)$. The role of 3D-speckle tracking has been investigated by Atsumi et al. [44]. 3D-speckle tracking records endocardial surface changes during the cardiac cycle and may provide more information than $2 \mathrm{D}$ longitudinal deformation. Both multiplane- and $3 \mathrm{D}$-speckle measurement of tracking are promising new applications in RV deformation imaging. However, further validation is needed before recommendations for clinical implementations can be made.

A major advantage of tissue deformation imaging compared to conventional echocardiographic wall motion analysis, is the ability to quantify regional wall motion rather than visual qualitative assessment. Quantifying wall motion facilitates the detection of subtle regional myocardial dysfunction. However, deformation imaging analysis is not hampered by visual misinterpretation of wall motion caused by tissue tethering. A third advantage is the ability to compare different myocardial segments over time to measure mechanical synchrony.

\section{Systolic deformation}

In this section, we provide the current published applications in deformation imaging.

As previously mentioned, fibrofatty replacement of the myocardium subsequently impairs wall motion at specific sites in the RV. This is reflected by a reduced amount of peak systolic strain in the RV free wall in ARVD/C patients compared to controls (Fig. 6) [20, 36, 45]. Peak systolic strain seems to be a reliable measurement of impaired wall motion and has high diagnostic value [17, 20, 42, 46, 47]. Moreover, deformation imaging reveals subtle wall motion abnormalities often in the absence of RV dilatation, global RV systolic function, and visual wall motion abnormalities [17]. Therefore, diagnostic performance of peak systolic strain was found to be superior to conventional measurements in ARVD/C patients [17, 42, 47]. A cut-off value of $-18 \%$ peak systolic strain, to differentiate between normal and abnormal RV segments, seems to be a reliable parameter and was reported by Prakasa et al. [47]. Diagnostic performance was measured by Teske et al. [17] who found sensitivity of $97 \%$ and a specificity of $91 \%$. Implementations of these findings (systolic peak strain and the presence of post-systolic shortening, in asymptomatic ARVD/C mutation carriers showed abnormalities in $71 \%$ of patients even though imaging criteria were not met by the $2010 \mathrm{TFC}$ ) [28]. While these measurements are very sensitive to detect regional contractility abnormalities, they lack specificity since other disease processes affecting the myocardium (ischemia, sarcoidosis, etc.) also produce a reduction of deformation parameters.

Beside regional wall motion analysis, deformation imaging also provides accurate measurements of global systolic RV function. In a (partial) ARVD/C cohort, mean RV free wall peak strain showed the strongest correlation with MRI obtained RVEF compared to other conventional 2D systolic parameters [48]. Several studies suggest an important role of exercise in the pathogenesis of ARVD/C $[49,50]$. Extensive exercise seems to accelerate the course of disease. Acute effect of exercise on cardiac function was explored with stress echocardiography by Vitarelli et al. [42]. The normal increase in both RV and LV global longitudinal shortening was reduced after exercise in ARVD/C patients compared to controls. This is most likely explained by the fact that higher loading conditions in the RV during exercise unmask abnormal segments that have normal function in the normal (low 
pressure - low volume) resting situation. Diagnostic performance of these parameters exceeded both conventional measurements and regional longitudinal strain parameters. Both sensitivity and specificity were reported as greater than $90 \%$ [42].

\section{Deformation after pulmonary valve closure}

Post-systolic shortening (PSS) is an additional deformation imaging-derived parameter frequently detected in ARVD/C [28]. PSS is defined as the presence of longitudinal shortening after semilunar valve closure (i.e. pulmonary valve) [51]. It is a well described phenomenon in both ischemic-and non-ischemic cardiomyopathies [51-53]. The exact mechanism of PSS is unclear. In a dyskinetic segment, PSS may originate as passive recoil by tissue interaction with surrounding contracting segments [45]. PSS could also be explained by local differences in electro-mechanical delay resulting in an out of phase active contraction [53]. Post-systolic shortening was found in $77.4 \%$ of the ARVD/C patients in the subtricuspid area [17]. Moreover, post-systolic shortening is proven to be an early sign of disease in asymptomatic PKP2 mutation carriers (64\%) without an established phenotypic expression of disease and could be of value in diagnosing ARVD/C (Fig. 6) [28].

\section{Dispersion of timing}

Evidence is accumulating that electrical abnormalities precede structural alterations in ARVD/C [5, 54]. Consequently, early phenotypic expressions of ARVD/C would likely be of electrical-rather than structural origin. Due to the electro-mechanical contraction coupling, delay in myocardial electrical activation could be studied by mechanical activation patterns [55-57]. The high temporal resolution of echocardiography makes deformation imaging a suitable tool for measuring mechanical dyssynchrony. Measurements of dyssynchrony could provide evidence of early, subtle electrical disturbances in ARVD/C, and may further improve the diagnostic value of echocardiography $[21,58]$. Moreover, due to the close relationship with electrical dyssynchrony, or electrical activation delay, mechanical dyssynchrony also contains possible prognostic information in predicting future arrhythmic events [21].

RV mechanical dyssynchrony in ARVD/C patients was first described by measuring time to peak velocity [58]. Tops et al. [58] showed marked delay in timing of septal and RV free wall peak velocity in ARVD/C compared to controls, indicating $\mathrm{RV}$ mechanical delay. Regional time to systolic peak data was analyzed by Sarvari et al. [21] and showed that dispersion of time to peak systolic value within the RV was markedly increased in $\mathrm{ARVD} / \mathrm{C}$ patients compared to controls. They also found this effect in asymptomatic ARVD/C mutation carriers not fulfilling $2010 \mathrm{TFC}$, indicating early diagnostic value of mechanical dyssynchrony. Increased mechanical dispersion, probably due to the close correlation with electrical dyssynchrony, was also correlated with the occurrence of ventricular arrhythmias in $\mathrm{ARVD} / \mathrm{C}$ patients [21].

\section{Discussion}

Echocardiography is a non-invasive imaging tool that has an established role in both diagnosis and prognosis of ARVD/C patients [12, 24]. Early and accurate diagnosis of ARVD/C is pivotal, since lethal ventricular arrhythmias may occur in the very early stages of ARVD/C before overt structural alterations appear [59]. The current $2010 \mathrm{TFC}$ echocardiographic measurements are not considered equally reliable as MRI based measurements [14]. Specifically, the difficult detection of regional wall abnormalities results in low sensitivity of echocardiography [14]. Several groups have shown the incremental diagnostic value of new echocardiographic tools such as 3D-echocardiography and tissue deformation imaging, and this could change the diagnostic role of echocardiography in ARVD/C.

Recent advances in 3D-echocardiography could improve assessment of RV volume and RV function and may prevent false-negative findings if performed together with other established conventional echocardiographic parameters [36]. Adding tissue deformation imaging derived parameters, especially peak systolic strain, could increase the yield of ARVD/C echocardiographic TFC [17, 20, 36, 46].

Recent insights into the pathophysiology of ARVD/C emphasize the importance of further exploring the parameters of mechanical dyssynchrony $[5,54]$. Mechanical activation parameters derived from tissue deformation imaging could be of incremental value, especially the early detection of ARVD/C [55-57].

Risk stratification in ARVD/C mutation carriers is hampered by the large variability of both penetrance and disease severity (phenotypic expression) during the course of the disease. MRI is often not feasible for the periodical follow-up of $\mathrm{ARVD} / \mathrm{C}$ patients due to the high rate of ICD implantation and the relative high cost. Thus, identifying reliable echocardiographic predictors in both early and advanced stages of disease is of 
utmost importance. Prognostic echocardiographic factors are already established in the more advanced disease stages [22, 32]. Both echocardiographic measured RV dysfunction and LV systolic dysfunction are known predictors for adverse outcome in ARVD/C [19, 31, 27]. However, life-threatening arrhythmic events may occur before extensive global systolic dysfunction. Future studies should focus on the correlation of subtle regional dysfunction and dyssynchrony with cardiac events [21, 59]. A study on the comparison between deformation imaging derived regional abnormalities and electrical abnormalities found during an electrophysiological study has to be performed to validate the current findings in electromechanical dyssynchrony. Validation is of particular importance since a recent study showed a weak correlation between late gadolinium contrast enhanced regions by MRI and the electro-anatomical scar a by electrophysiological study [60].

Finally, subtle changes in volumes and function over time could also have incremental value in predicting cardiac events compared to conventional measurements.

\section{Conclusions}

Conventional echocardiography has an established role in diagnosis and prognosis of ARVD/C. Novel echocardiographic parameters, derived from 3D-RV echocardiography and RV tissue deformation imaging, are promising to improve diagnostic sensitivity and specificity. Further validation is mandatory. Nevertheless, new echocardiographic parameters, combined with conventional parameters, enhances detection of phenotypic expressions of $\mathrm{ARVD} / \mathrm{C}$ and indicates that there is a current and future role for echocardiography in ARVD/C.

In the ARVD/C patient, both echocardiography and MRI will remain complementary imaging modalities in the near future. Novel validated echocardiographic parameters will have the capability to detect subtle regional changes for the purpose of early diagnosis in absence of global RV structural alterations. Serial echocardiographic examination will provide insight in the progression of $\mathrm{ARVD} / \mathrm{C}$. Identification of robust predictors of adverse clinical outcome will guide therapeutic strategies and may prevent life threatening events.

\section{Acknowledgements}

We thank Dr. Folkert J. Meijboom and Bastiaan A. Mast for providing their figures and artwork.

Conflict of interest: None declared

\section{References}

1. Marcus FI, Fontaine GH, Guiraudon G et al. Right ventricular dysplasia: A report of 24 adult cases. Circulation, 1982; 65: 384-398.

2. Sen-Chowdhry S, Syrris P, McKenna WJ. Role of genetic analysis in the management of patients with arrhythmogenic right ventricular dysplasia/cardiomyopathy. J Am Coll Cardiol, 2007; 50: 1813-1821.

3. Cox MG, van der Zwaag PA, van der Werf $\mathrm{C}$ et al. Arrhythmogenic right ventricular dysplasia/cardiomyopathy: Pathogenic desmosome mutations in index-patients predict outcome of family screening: Dutch arrhythmogenic right ventricular dysplasia/ /cardiomyopathy genotype-phenotype follow-up study. Circulation, 2011; 123: 2690-2700.

4. Noorman M, van der Heyden MA, van Veen TA et al. Cardiac cell-cell junctions in health and disease: Electrical versus mechanical coupling. J Mol Cell Cardiol, 2009; 47: 23-31.

5. Cerrone M, Noorman M, Lin X et al. Sodium current deficit and arrhythmogenesis in a murine model of plakophilin-2 haploinsufficiency. Cardiovasc Res, 2012; 95: 460-468.

6. Kim C, Wong J, Wen J et al. Studying arrhythmogenic right ventricular dysplasia with patient-specific iPSCs. Nature, 2013; 494: 105-110.

7. de Bakker JM, van Capelle FJ, Janse MJ et al. Slow conduction in the infarcted human heart. 'Zigzag' course of activation. Circulation, 1993; 88: 915-926.

8. Dalal D, Molin LH, Piccini J et al. Clinical features of arrhythmogenic right ventricular dysplasia/cardiomyopathy associated with mutations in plakophilin-2. Circulation, 2006; 113: 1641-1649.

9. Fressart V, Duthoit G, Donal E et al. Desmosomal gene analysis in arrhythmogenic right ventricular dysplasia/cardiomyopathy: Spectrum of mutations and clinical impact in practice. Europace, 2010; 12: 861-868.

10. Te Riele AS, James CA, Philips B, et al. Mutation-positive arrhythmogenic right ventricular dysplasia/cardiomyopathy: The triangle of dysplasia displaced. J Cardiovasc Electrophysiol, 2013; 24: 1311-1320.

11. Lindstrom L, Nylander E, Larsson H, Wranne B. Left ventricular involvement in arrhythmogenic right ventricular cardiomyopathy: A scintigraphic and echocardiographic study. Clin Physiol Funct Imaging, 2005; 25: 171-177.

12. Marcus FI, McKenna WJ, Sherrill D et al. Diagnosis of arrhythmogenic right ventricular cardiomyopathy/dysplasia: Proposed modification of the task force criteria. Circulation, 2010; 121: 1533-1541.

13. Tandri H, Macedo R, Calkins H et al. Role of magnetic resonance imaging in arrhythmogenic right ventricular dysplasia: Insights from the North American arrhythmogenic right ventricular dysplasia (ARVD/C) study. Am Heart J, 2008; 155: 147-153.

14. Borgquist R, Haugaa KH, Gilljam T et al. The diagnostic performance of imaging methods in ARVC using the 2010 Task Force criteria. Eur Heart J Cardiovasc Imaging, 2014; 15: 1219-1225.

15. Bomma C, Rutberg J, Tandri H et al. Misdiagnosis of arrhythmogenic right ventricular dysplasia/cardiomyopathy. J Cardiovasc Electrophysiol, 2004; 15: 300-306.

16. Sievers B, Addo M, Franken U, Trappe HJ. Right ventricular wall motion abnormalities found in healthy subjects by cardiovascular magnetic resonance imaging and characterized with a new segmental model. J Cardiovasc Magn Reson, 2004; 6: 601-608. 
17. Teske AJ, Cox MG, De Boeck BW, Doevendans PA, Hauer RN, Cramer MJ. Echocardiographic tissue deformation imaging quantifies abnormal regional right ventricular function in arrhythmogenic right ventricular dysplasia/cardiomyopathy. J Am Soc Echocardiogr, 2009; 22: 920-927.

18. Teske AJ, De Boeck BW, Melman PG, Sieswerda GT, Doevendans PA, Cramer MJ. Echocardiographic quantification of myocardial function using tissue deformation imaging, a guide to image acquisition and analysis using tissue Doppler and speckle tracking. Cardiovasc Ultrasound, 2007; 5: 27.

19. Prakasa KR, Dalal D, Wang J et al. Feasibility and variability of three dimensional echocardiography in arrhythmogenic right ventricular dysplasia/cardiomyopathy. Am J Cardiol, 2006; 97: 703-709.

20. Kjaergaard J, Hastrup Svendsen J, Sogaard P et al. Advanced quantitative echocardiography in arrhythmogenic right ventricular cardiomyopathy. J Am Soc Echocardiogr, 2007; 20: 27-35.

21. Sarvari SI, Haugaa KH, Anfinsen OG et al. Right ventricular mechanical dispersion is related to malignant arrhythmias: A study of patients with arrhythmogenic right ventricular cardiomyopathy and subclinical right ventricular dysfunction. Eur Heart J, 2011; 32: 1089-1096.

22. Saguner AM, Vecchiati A, Baldinger SH et al. Different prognostic value of functional right ventricular parameters in arrhythmogenic right ventricular cardiomyopathy/dysplasia. Circ Cardiovasc Imaging 2014; 7: 230-239.

23. Blomstrom-Lundqvist C, Beckman-Suurkula M, Wallentin I, Jonsson R, Olsson SB. Ventricular dimensions and wall motion assessed by echocardiography in patients with arrhythmogenic right ventricular dysplasia. Eur Heart J, 1988; 9: 1291-1302.

24. Yoerger DM, Marcus F, Sherrill D et al. Echocardiographic findings in patients meeting task force criteria for arrhythmogenic right ventricular dysplasia: new insights from the multidisciplinary study of right ventricular dysplasia. J Am Coll Cardiol, 2005; 45: 860-865.

25. Lindstrom L, Wilkenshoff UM, Larsson H, Wranne B. Echocardiographic assessment of arrhythmogenic right ventricular cardiomyopathy. Heart, 2001; 86: 31-38.

26. Rudski LG, Lai WW, Afilalo J et al. Guidelines for the echocardiographic assessment of the right heart in adults: a report from the American Society of Echocardiography endorsed by the European Association of Echocardiography, a registered branch of the European Society of Cardiology, and the Canadian Society of Echocardiography. J Am Soc Echocardiog, 2010; 23: 685-713.

27. Wang J, Prakasa K, Bomma C et al. Comparison of novel echocardiographic parameters of right ventricular function with ejection fraction by cardiac magnetic resonance. J Am Soc Echocardiogr, 2007; 20: 1058-1064.

28. Teske AJ, Cox MG, Te Riele AS et al. Early detection of regional functional abnormalities in asymptomatic ARVD/C gene carriers. J Am Soc Echocardiogr, 2012; 25: 997-1006.

29. Foale R, Nihoyannopoulos P, McKenna W et al. Echocardiographic measurement of the normal adult right ventricle. $\mathrm{Br}$ Heart J, 1986; 56: 33-44.

30. Tosoratti E, Badano LP, Gianfagna P et al. Improved delineation of morphological features of arrhythmogenic right ventricular cardiomyopathy with the use of contrast-enhanced echocardiography. J Cardiovasc Med (Hagerstown), 2006; 7: 566-568.

31. Lemola K, Brunckhorst C, Helfenstein U, Oechslin E, Jenni R, Duru F. Predictors of adverse outcome in patients with ar- rhythmogenic right ventricular dysplasia/cardiomyopathy: Long term experience of a tertiary care centre. Heart, 2005; 91: 1167-1172.

32. Pinamonti B, Dragos AM, Pyxaras SA et al. Prognostic predictors in arrhythmogenic right ventricular cardiomyopathy: Results from a 10-year registry. Eur Heart J, 2011; 32: 1105-1113.

33. Sen-Chowdhry S, Syrris P, Ward D, Asimaki A, Sevdalis E, McKenna WJ. Clinical and genetic characterization of families with arrhythmogenic right ventricular dysplasia/cardiomyopathy provides novel insights into patterns of disease expression. Circulation, 2007; 115: 1710-1720.

34. Teske AJ, Cox MG, Peterse MC, Cramer MJ, Hauer RN. Case report: Echocardiographic deformation imaging detects left ventricular involvement in a young boy with arrhythmogenic right ventricular dysplasia/cardiomyopathy. Int J Cardiol, 2009; 135: e24-e26.

35. Knight DS, Grasso AE, Quail MA et al. Accuracy and reproducibility of right ventricular quantification in patients with pressure and volume overload using single-beat three-dimensional echocardiography. J Am Soc Echocardiogr, 2015; 28: 363-374.

36. Kjaergaard J. Assessment of right ventricular systolic function by tissue Doppler echocardiography. Dan Med J, 2012; 59: B4409.

37. Bhave NM, Patel AR, Weinert L et al. Three-dimensional modeling of the right ventricle from two-dimensional transthoracic echocardiographic images: Utility of knowledge-based reconstruction in pulmonary arterial hypertension. J Am Soc Echocardiogr, 2013; 26: 860-867.

38. Dragulescu A, Grosse-Wortmann L, Fackoury C, Mertens L. Echocardiographic assessment of right ventricular volumes: A comparison of different techniques in children after surgical repair of tetralogy of Fallot. Eur Heart J Cardiovasc Imaging, 2012; 13: 596-604.

39. Kannan A, Poongkunran C, Jayaraj M, Janardhanan R. Role of strain imaging in right heart disease: a comprehensive review. J Clin Med Res, 2014; 6: 309-313.

40. Carlsson M, Ugander M, Heiberg E, Arheden H. The quantitative relationship between longitudinal and radial function in left, right, and total heart pumping in humans. Am J Physiol Heart Circ Physiol, 2007; 293: H636-H644.

41. Gustafsson U, Lindqvist P, Waldenstrom A. Apical circumferential motion of the right and the left ventricles in healthy subjects described with speckle tracking. J Am Soc Echocardiogr, 2008; 21: 1326-1330.

42. Vitarelli A, Cortes Morichetti M, Capotosto L et al. Utility of strain echocardiography at rest and after stress testing in arrhythmogenic right ventricular dysplasia. Am J Cardiol 2013; 111: 1344-1350.

43. Greiner S, Heimisch M, Aurich M, Hess JA, Katus HA, Mereles D. Multiplane two-dimensional strain echocardiography for segmental analysis of right ventricular mechanics: New-RV study. Clin Res Cardiol, 2014; 103: 817-824.

44. Atsumi A, Ishizu T, Kameda Y et al. Application of 3-dimensional speckle tracking imaging to the assessment of right ventricular regional deformation. Circ J, 2013; 77: 1760-1768.

45. Herbots L, Kowalski M, Vanhaecke J, Hatle L, Sutherland GR. Characterizing abnormal regional longitudinal function in arrhythmogenic right ventricular dysplasia. The potential clinical role of ultrasonic myocardial deformation imaging. Eur J Echocardiogr, 2003; 4: 101-107.

46. Aneq MA, Engvall J, Brudin L, Nylander E. Evaluation of right and left ventricular function using speckle tracking echocardiography 
in patients with arrhythmogenic right ventricular cardiomyopathy and their first degree relatives. Cardiovasc Ultrasound 2012; 10: 37.

47. Prakasa KR, Wang J, Tandri H et al. Utility of tissue Doppler and strain echocardiography in arrhythmogenic right ventricular dysplasia/cardiomyopathy. Am J Cardiol, 2007; 100: 507-512.

48. Focardi M, Cameli M, Carbone SF et al. Traditional and innovative echocardiographic parameters for the analysis of right ventricular performance in comparison with cardiac magnetic resonance. Eur Heart J Cardiovasc Imaging, 2015; 16: 47-52.

49. Sawant AC, Bhonsale A, Te Riele AS et al. Exercise has a disproportionate role in the pathogenesis of arrhythmogenic right ventricular dysplasia/cardiomyopathy in patients without desmosomal mutations. J Am Heart Assoc, 2014; 3: e001471. doi: 10.1161/JAHA.114.001471.

50. James CA, Bhonsale A, Tichnell C et al. Exercise increases agerelated penetrance and arrhythmic risk in arrhythmogenic right ventricular dysplasia/cardiomyopathy-associated desmosomal mutation carriers. J Am Coll Cardiol, 2013; 62: 1290-1297.

51. Weidemann F, Niemann M, Herrmann S et al. A new echocardiographic approach for the detection of non-ischaemic fibrosis in hypertrophic myocardium. Eur Heart J, 2007; 28: 3020-3026.

52. Skulstad H, Edvardsen T, Urheim S et al. Postsystolic shortening in ischemic myocardium: Active contraction or passive recoil? Circulation, 2002; 106: 718-724.

53. HuiW,SlorachC, DragulescuA, Mertens L, Bijnens B, FriedbergMK. Mechanisms of right ventricular electromechanical dyssynchro- ny and mechanical inefficiency in children after repair of tetralogy of fallot. Circ Cardiovasc Imaging, 2014; 7: 610-618.

54. te Riele AS, James CA, Rastegar N et al. Yield of serial evaluation in at-risk family members of patients with ARVD/C. J Am Coll Cardiol, 2014; 64: 293-301.

55. De Boeck BW, Teske AJ, Leenders GE et al. Detection and quantification by deformation imaging of the functional impact of septal compared to free wall preexcitation in the Wolff-Parkinson-White syndrome. Am J Cardiol, 2010; 106: 539-546.e532.

56. Prinzen FW, Augustijn CH, Allessie MA, Arts T, Delhaas T, Reneman RS. The time sequence of electrical and mechanical activation during spontaneous beating and ectopic stimulation. Eur Heart J, 1992; 13: 535-543.

57. Wyman BT, Hunter WC, Prinzen FW, McVeigh ER. Mapping propagation of mechanical activation in the paced heart with MRI tagging. Am J Physiol, 1999;276: H881-H891.

58. Tops LF, Prakasa K, Tandri H et al. Prevalence and pathophysiologic attributes of ventricular dyssynchrony in arrhythmogenic right ventricular dysplasia/cardiomyopathy. J Am Coll Cardiol, 2009; 54: 445-451.

59. Corrado D, Basso C, Thiene G. Sudden cardiac death in young people with apparently normal heart. Cardiovasc Res, 2001; 50: 399-408.

60. Marra MP, Leoni L, Bauce B et al. Imaging study of ventricular scar in arrhythmogenic right ventricular cardiomyopathy: comparison of 3D standard electroanatomical voltage mapping and contrast-enhanced cardiac magnetic resonance. Circ Arrhythm Electrophysiol, 2012; 5: 91-100. 